\title{
Cryopreservation of Hevea brasiliensis zygotic embryos by vitrification and encapsulation-dehydration
}

\author{
Korakot Nakkanong • Charassri Nualsri
}

Received: 12 March 2018 / Revised: 19 June 2018 / Revised: 3 July 2018 / Revised: 9 July 2018 / Accepted: 10 July 2018

(C) Korean Society for Plant Biotechnology

\begin{abstract}
The mature zygotic embryos of the Hevea brasiliensis were cryopreserved through the use of the vitrification and encapsulation/dehydration techniques. In all the experiments, the zygotic embryos were pre-cultured for three days in the MS medium supplemented with $0.3 \mathrm{M}$ sucrose before they were used for the cryopreservation technique. In the vitrification procedure, the effect of the plant vitrification solutions (PVS2 and PVS3) and exposure time were studied. The highest survival rate $(88.87 \%)$ and regrowth $(66.33 \%)$ were achieved when the precultured zygotic embryos were incubated in a loading solution for 20 minutes at $0^{\circ} \mathrm{C}$. They were subsequently exposed to PVS2 for 120 minutes at $0^{\circ} \mathrm{C}$ and plunged directly into liquid nitrogen. Cryopreservation by the encapsulation-dehydration method was successfully done by leaving the encapsulated zygotic embryos in a laminar flow for 4 hours prior to plunging into a $L N$. The survival rate and regrowth of the encapsulated zygotic embryos were $37.50 \%$ and $27.98 \%$, respectively. The cryopreserved zygotic embryos were able to develop into whole plants.
\end{abstract}

Keywords zygotic embryo, Hevea brasiliensis, cryopreservation, vitrification, encapsulation-dehydration

\section{Introduction}

Cryopreservation is becoming a very important tool for the long-term storage of plant genetic resources and efficient cryopreservation protocols have been developed for a large number of plant species. Conservation of Hevea brasiliensis genetic resources is presently through field banks. However,

K. Nakkanong $(\bowtie) \cdot$ C. Nualsri

Department of Plant Science, Faculty of Natural Resources, Prince of Songkla University, Hat Yai, Songkhla, 90112, Thailand e-mail: korakot_nick@yahoo.com field conservation can be endangered by many field factors, such as pest and disease outbreak, besides competing for limited land resources for other development. Furthermore, Hevea seeds are difficult to conserve as they are recalcitrant seed. Seed viability drops as soon as fruits are collected. Charloq et al. (2016) reported that during storage by avoid sunlight exposer, the seed germination will decrease to $0 \%$ after 14 days. The only practicable procedure for long-term storage of rubber tree germplasm is cryopreservation, however this requires partial drying to prevent ice crystal damage. Previous studied on Hevea zygotic embryos cryopreservation was reported by Normah et al. (1986). The highest percentages of survival rate $(69 \%)$ was obtained when excised zygotic embryos were desiccated for $3 \mathrm{~h}$, directly plunging into liquid nitrogen (LN) and rapidly thawed. Yap et al. (1998) reported that preculture of excised embryos in 0.3 $M$ sucrose improved desiccation and freezing tolerance resulting in higher viability and survival rate after cryopreservation. Additionally, Hevea cryopreservation of anther callus by vitrification showed $71.7 \%$ viability. This effective protocol involved preculture on MS medium containing 5\% sucrose $(\mathrm{w} / \mathrm{v})$ and $5 \%$ dimethyl sulfoxide (DMSO) $(\mathrm{v} / \mathrm{v})$ for 3 days, loading with $60 \%$ PVS2 for $20 \mathrm{~min}$ at $0^{\circ} \mathrm{C}$ and dehydrate with ice cold PVS2 for $40 \mathrm{~min}$ (Zhou et al. 2012). However, only moderate survival rate and a low percentage of embryos developed into normal shoots were observed. Therefore, the further improvement should be carried out to enhance survival and regrowth rate after cryopreservation.

Different techniques are used for cryopreservation of recalcitrant seed species. New cryopreservation protocols based on vitrification as well as encapsulation-dehydration have been developed. Vitrification relies on treatment of explants with a concentrated vitrification solution for variable periods of time (from 15 minutes up to 6 hours), followed by a direct plunge into liquid nitrogen. The success of the procedure can be attributed to its easiness, high reproduc- 
ibility and to the fact that it can successfully be applied to a wide range of tissues and plant species (Wang et al. 2005; Sajini et al. 2011). Additionally, encapsulation-dehydration technique was established by Fabre and Dereuddre (1990), explants (usually meristems or embryos) are firstly encapsulated in alginate beads, then the encapsulated explants are treated with a high sucrose concentration, dried down to a moisture content of $20 \sim 30 \%$ (under airflow or using silica gel) and subsequently rapidly frozen in liquid nitrogen. Although the procedure can be considered rather lengthy and labour-intensive. However, it is observed that the presence of a nutritive matrix (the bead) surrounding the explant can promote its regrowth after rewarming. These protocols, which have proved its efficiency with various plant species, have not been tested yet with Hevea zygotic embryos. For successful cryopreservation, many factors are involved, such as starting materials, pretreatment conditions, cryopreservation procedures and post-thaw treatment. In our experiments, we compared vitrification and encapsulation-dehydration using zygotic embryos of rubber tree. For vitrification protocol, osmotic dehydration was performed with the two most generally used plant vitrification solutions, PVS2 (Sakai et al. 1990) and PVS3 (Nishizawa et al. 1993). Moreover, encapsulation-dehydration by drying under airflow or using silica gel was compared. Therefore the goals of this study were to investigate the potential of long-term conservation (cryopreservation) by the application of vitrification and encapsulation-dehydration method to define a reliable method for rubber tree germplasm conservation.

\section{Materials and Methods}

\section{Plant materials}

Hevea clones used in this studied were early introduced clone which collected from Southern Thailand. Zygotic embryos of these clone were excised from the mature seeds and subsequently disinfection followed the method of Ighere et al. (2011). The seed testa was removed and the cotyledon was washed in running tap water for $2 \mathrm{~min}$. The explants were further washed with tween 20 before they were immersed in $70 \%$ ethanol for $5 \mathrm{~min}$, thereafter the explants were rinsed with sterile distilled water. Sodium hypochlorite solution ( $2 \%$ active chlorine) was used for the next disinfection (15 min immersion). After washing with sterile distilled water, further disinfection was done using sodium hypochlorite solution ( $1 \%$ active chlorine) for $10 \mathrm{~min}$. The explants were then rinsed with sterile distilled water for five times. All disinfection process was carried out in the laminar flow hood. Zygotic embryos were large with a diameter of $2 \sim 3 \mathrm{~mm}$ and a length of $4 \sim 6 \mathrm{~mm}$. Embryos were excised and maintained on Murashige and Skoog (MS) media for 5 days and viable pale yellow explants were used for the further steps. The samples were placed under the light intensity of 2,500 lux, 12 hour photoperiod, $25^{\circ} \mathrm{C}$.

\section{Vitrification}

\section{Optimization of sucrose pretreatment duration}

Preculture of explants was done on semi-solid MS media supplemented with progressive concentrations of sucrose $(0,0.1,0.3,0.5,0.7$ and $1.0 \mathrm{M})$ in Petri dishes for 3 day (preliminary study). The optimum time for sucrose pretreatment $0,1,2,3,4$ and 5 days were tested. Precultured zygotic embryos were used for the following vitrification method

\section{Cryoprotection duration with PVS2 and PVS3}

Two most generally used plant vitrification solutions (Sakai et al. 1991), PVS2 [30\% (w/v) glycerol, 15\% (w/v) ethylene glycol, 15\% (w/v) dimethyl sulphoxide (DMSO)] and PVS3 [50\% sucrose and $50 \%$ glycerol] were compered. Moreover, an experiment was carried out to determine the optimal PVS2 and PVS3 exposure time. Zygotic embryos were incubated in loading solution (MS medium with $2 \mathrm{M}$ glycerol and $0.4 \mathrm{M}$ sucrose) for $20 \mathrm{~min}$ at $0^{\circ} \mathrm{C}$. The loading solution was then removed and subsequently subjected to dehydration with PVS2 and PVS3. The zygotic embryos were exposed to the vitrification solutions for varying times $(0,30,60,90$ and $120 \mathrm{~min})$ at $0^{\circ} \mathrm{C}$. Cryotubes containing the zygotic embryos were plunged directly in $\mathrm{LN}$ and maintained at $-196^{\circ} \mathrm{C}$ for $24 \mathrm{~h}$. After storage in $\mathrm{LN}$, cryotubes were rewarmed rapidly in a water bath at $40 \pm$ $2^{\circ} \mathrm{C}$ for $5 \mathrm{~min}$. Subsequently, the vitrification solution was drained from the cryotubes and replaced with unloading solution (MS liquid medium supplemented with $1.2 \mathrm{M}$ sucrose), in which the zygotic embryos were washed for $20 \mathrm{~min}$. Then zygotic embryos were transferred to regrowth medium (MS medium supplemented with $0.6 \sim 0.7 \mu \mathrm{M}$ kinetin $(\mathrm{KN}), 1.0 \mu \mathrm{M}$ naphthaleneacetic acid (NAA), $1.4 \mu \mathrm{M}$ Gibberellic acid (GA) and $4 \mathrm{~g} / 1$ activated charcoal). The cultures were placed under the light intensity of 2,500 lux, 12 hour photoperiod, $25^{\circ} \mathrm{C}$. Vitrificated but unfrozen beads were used as controls (-LN). 


\section{Encapsulation-dehydration}

Excised zygotic embryos were encapsulated according to the method of Peran et al. (2006). Zygotic embryos were suspended in calcium-free medium supplemented with $3 \%$ sodium alginate. Calcium alginate beads were formed by dispensing drops of $3 \%$ sodium alginate each containing a single embryonic axis (diameter $4 \sim 5 \mathrm{~mm}$ ), into a $0.3 \mathrm{M}$ $\mathrm{CaCl}_{2}$ solution. After preculture treatment with $0.3 \mathrm{M}$ sucrose for 3 days, encapsulated zygotic embryos were dried at $25^{\circ} \mathrm{C}$ either by rapidly flash drying or slowly by using silica gel. Flash drying was accomplished by placing encapsulated zygotic embryos on top of a petri dish, gradually dehydrated (0-5 h) by exposure to the sterile air flow. For slow drying, zygotic embryos were placed in closed containers (10 beads per container) filled with $80 \mathrm{~g}$ silica gel. Moisture content of the beads after each dehydration period was calculated on fresh weight basis. Treated explants were then placed in sterilized aluminium foil enveloped and directly dipped in LN and kept for a minimum of $24 \mathrm{~h}$. Dehydrated but unfrozen beads were used as controls (-LN). Cryopreserved axes were thawed rapidly by immersing the aluminium foil envelops in water bath at $40 \pm 2^{\circ} \mathrm{C}$ for $5 \mathrm{~min}$ subsequently plating on regrowth medium.

\section{Assessment of survival rate and regrowth percentage}

The effect of different treatments was assessed by measuring survival rate and regrowth percentages of zygotic embryos. Survival rate in corresponding to the presence of living tissues and to the observation of any regrowth pattern was recorded after 10 days of cryopreservation. After this duration, dead embryogenic axes normally changed into pale white color. Measuring of survival rate was important as it allowed rapid evaluation of experiments performed. Regrowth was evaluated from the production of normal roots and shoots from treated explants which observed after 20 days of culture. Each treatment was performed with three replicates of 10 explants. The results were based on the mean value of at least three sets of independent experiment, presented as percentage of survival rate/regrowth samples over the total number of explants treated per experimental condition.

\section{Statistical analysis}

All experiments were repeated three times. Results are presented as mean percentage with their standard error, and evaluated by SPSS 19 and Excel software. Significance difference among mean values was assessed by analysis of variance (ANOVA) and Duncan's multiple range test $(\mathrm{P}<0.05)$.

\section{Results and Discussion}

\section{Vitrification}

\section{Duration of sucrose preculture}

To induce dehydration tolerance, explants need some treatments before immerging in LN, such as preculture on medium with a high sucrose concentration. The injurious effects caused by the dehydration process are reduced by optimizing the sucrose concentration in the preculture medium and the duration of preculture step. In preliminary study on effect of progressive sucrose concentration in the preculture medium showed that the viable rate of zygotic embryos after cooling in LN increased with sucrose concentration in preculture medium, peaked $(66.2 \%)$ at $0.3 \mathrm{M}$ and progressively decreased to $52.7 \%$ when the sucrose concentration was raised to $1.0 \mathrm{M}$ (data not shown). The effect of extending the preculture duration in medium with $0.3 \mathrm{M}$ sucrose was also studied. As showed in Figure 1, after 3 days of preculture, the highest survival rate was achieved, while preculture for 4-5 days decreased the survival rate.

The prolonging of preculture time, the viability of zygotic embryos dramatically increased and reached a maximum at 3 days. Survival rate and regrowth percentage after LN



Fig. 1 The effect of sucrose concentration in the preculture medium on the regeneration of the cryopreserved rubber tree zygotic embryos by dehydration. Bars correspond to the SE of means of three replications. Values with different letters were significantly different using Duncan's Multiple Range Test $(\mathrm{P}<$ 0.05 ) 
exposures were 75.5 and $62.3 \%$. Our results showed that sucrose preculture can enhance dessication and freezing tolerance of rubber zygotic embryos. Viable zygotic embryos turned to green within $7 \sim 10$ days after culture on regrowth medium. Growth of cryopreserved zygotic embryos showed normal shoots and roots emergence. However the development of shoots and roots was slower than the control seedling (no cryopreservation). Survival rate of control zygotic embryos was consistently above $90 \%$, whatever the sucrose concentration employed during pretreatment. In accordance with Yap et al. (2011) who investigated the effects of sucrose preculture duration on tolerance of Garcinia cowa shoot tips to cryopreservation using the PVS2 vitrification solution. The result demonstrated that increasing preculture duration on $0.3 \mathrm{M}$ sucrose medium from 0 to 3 days enhanced tolerance to PVS2 solution from 5.6\% (no preculture) to $49.2 \%$ (3 day preculture). However, the optimal protocol for preculture conditions of coconut zygotic embryos involved preculture of embryos for 3 days on semi-solid medium with $0.6 \mathrm{M}$ sucrose (Sajini et al. 2011). Uemura and Steponkus (2003) reported that exogenous sucrose at low concentration serves as a metabolic substrate for lowtemperature induced metabolic alterations, while at higher concentration it has a direct cryoprotective effect on cellular membranes. Thus, we used zygotic embryos precultured with $0.3 \mathrm{M}$ sucrose for 3 days in the following vitrification experiments.

\section{Cryoprotection duration with PVS2 and PVS3}

We compared the most widely used vitrification solutions, PVS2 and PVS3, which were employed for different durations. PVS2 is characterized by its high chemical toxicity, because it includes the permeating cryoprotectants DMSO and ethylene glycol, while PVS3, which includes sucrose and glycerol, can be toxic because of the high osmotic pressure it exerts on plant cells (Kim et al. 2009). In the preliminary experiments, it was observed that loading solution for $20 \mathrm{~min}$ at $0^{\circ} \mathrm{C}$ proved to be most effective in increasing survival rate of zygotic embryos cooled to $196^{\circ} \mathrm{C}$. To determine the optimum time of exposure to PVS2 and PVS3 solution at $0^{\circ} \mathrm{C}$, precultured zygotic embryos were dehydrated with PVS2 or PVS3 solution for different lengths of time prior to a plunge into LN. Exposure to PVS2 or PVS3 solution for various lengths of time resulted in a variable rate of survival rate and regrowth percentage (Table 1). The duration of PVS3 treatment is critical for regrowth of embryos after cryopreservation. In this study, longer exposure time for PVS3 treatments might be necessary since Sajini et al.(2011) demonstrated that the optimal PVS3 treatments for coconut zygotic embryos was achieved after $16 \mathrm{~h}$. A similar pattern was observed in garlic shoot tips treated with PVS3 for 150 $180 \mathrm{~min}$ ensured $92 \%$ regeneration after freezing (Kim et al. 2004). Moreover, our result shows that Hevea embryos were sensitive to osmotic stress induced by PVS3. Zygotic embryos treated with PVS2 solution at $0^{\circ} \mathrm{C}$ for up to $120 \mathrm{~min}$ without cooling (treated control) retained very high levels of survival rate and regrowth percentage. After treatment with PVS2, survival and regrowth percentage of precultured zygotic embryos increased progressively with increasing treatment durations (Table 1). After cryopreservation, the highest level of survival rate and regrowth percentage were obtained with precultured zygotic embryos treated with PVS2 for 120 min at $88.87 \%$ and $66.33 \%$, respectively. Similar results of using PVS2 exposure range ( $40 \sim 90 \mathrm{~min})$, were obtained in avocado (Guzmán-García et al. 2013), Quercus (Martí

Table 1 The survival rate and regrowth percentage of the vitrificated control (-LN) and cryopreserved (+LN) zygotic embryos

\begin{tabular}{|c|c|c|c|c|c|}
\hline \multirow{2}{*}{$\begin{array}{l}\text { Plant vitrification } \\
\text { solution }\end{array}$} & \multirow{2}{*}{$\begin{array}{l}\text { Length of exposure } \\
\text { (min) }\end{array}$} & \multicolumn{2}{|c|}{$+\mathrm{LN}$} & \multicolumn{2}{|c|}{$-\mathrm{LN}$} \\
\hline & & Survival rate $(\%)$ & Regrowth (\%) & Survival rate $(\%)$ & Regrowth (\%) \\
\hline \multirow[t]{5}{*}{ PVS2 } & 0 & 0 & 0 & $93.33 \pm 8.7^{\mathrm{a}}$ & $93.33 \pm 5.6^{\mathrm{a}}$ \\
\hline & 30 & 0 & 0 & $93.33 \pm 9.8^{\mathrm{a}}$ & $93.33 \pm 15.3^{\mathrm{a}}$ \\
\hline & 60 & $55.55 \pm 11.6^{\mathrm{c}}$ & $33.37 \pm 8.2^{\mathrm{c}}$ & $96.67 \pm 10.5^{\mathrm{a}}$ & $96.67 \pm 9.7^{\mathrm{a}}$ \\
\hline & 90 & $66.67 \pm 9.1^{\mathrm{b}}$ & $53.33 \pm 17.7^{\mathrm{b}}$ & $93.33 \pm 12.5^{\mathrm{a}}$ & $90.00 \pm 10.7^{\mathrm{a}}$ \\
\hline & 120 & $88.87 \pm 14.2^{\mathrm{a}}$ & $66.33 \pm 9.4^{\mathrm{a}}$ & $96.67 \pm 9.3^{\mathrm{a}}$ & $93.33 \pm 9.5^{\mathrm{a}}$ \\
\hline \multirow[t]{5}{*}{ PVS3 } & 0 & 0 & 0 & $93.33 \pm 5.7^{\mathrm{a}}$ & $93.33 \pm 8.7^{\mathrm{a}}$ \\
\hline & 30 & 0 & 0 & $83.33 \pm 6.3^{\mathrm{a}}$ & $83.33 \pm 7.5^{\mathrm{a}}$ \\
\hline & 60 & 0 & 0 & $86.67 \pm 9.5^{\mathrm{a}}$ & $86.67 \pm 13.4^{\mathrm{a}}$ \\
\hline & 90 & $23.33 \pm 7.7^{\mathrm{d}}$ & $16.67 \pm 5.7^{\mathrm{d}}$ & $83.33 \pm 12.3^{\mathrm{a}}$ & $80.00 \pm 15.7^{\mathrm{a}}$ \\
\hline & 120 & $33.33 \pm 9.4^{\mathrm{d}}$ & $30.00 \pm 10.2^{\mathrm{c}}$ & $86.67 \pm 14.8^{\mathrm{a}}$ & $86.67 \pm 17.3^{\mathrm{a}}$ \\
\hline
\end{tabular}

Values (mean values \pm SE) within the column of each parameter for each treatment followed by the same letter were not significantly different based on the Duncan's multiple range test $(\mathrm{P}<0.05)$ 


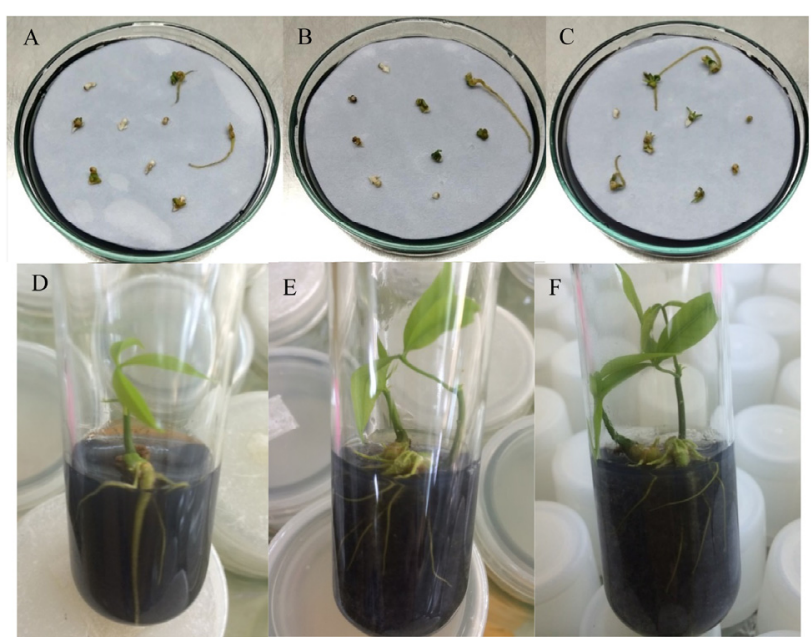

Fig. 2 Post-cryopreservation survival of the zygotic embryos by vitrification expose in PVS2 for 60 mins (A), 90 mins (B) and 120 mins (C), and the development of the zygotic embryos after 2 months of culturing on the regrowth medium (D-F)

nez et al. 2003) and taro (Sant et al. 2008), with recovery rates ranging from 77.78 to $100 \%$. Finding the adequate exposure time to PVS2 solution is a critical step in all vitrification procedures (Sakai and Engelmann 2007) as it is the key to find a balance between appropriate dehydration and chemical toxicity. A possible reason for this successful method could be the exposure of zygotic embryos to PVS2 at $0^{\circ} \mathrm{C}$ instead of $25^{\circ} \mathrm{C}$. Most injury effects caused by the dehydration process are reduced or eliminated by the loading step and optimizing PVS2 exposure at $0^{\circ} \mathrm{C}$ (Sakai et al 1990). Not only PVS2 exposure at $0^{\circ} \mathrm{C}$ gives better replication of results, but also a broad range of optimum PVS2 exposure time offers the practical advantage of cryopreserving larger numbers of explants in a day. This was also observed for banana where optimal length of PVS2 treatment was $30 \sim 50 \mathrm{~min}$ at $0^{\circ} \mathrm{C}$ (Panis et al. 2005). According to our results, $120 \mathrm{~min}$ can be proposed as the optimum PVS2 incubation time for rubber zygotic embryos as it is the balanced time point of this plant material. Nevertheless, certain toxicity could be attributed to LS and PVS2 solutions. Toxicity to LS solution was specific to Hevea zygotic embryos in which it provoked a significant decrease in minimum recovery (Sam 1999). In the present study, it was shown that the treated zygotic embryos by vitrification method delayed by 20 days compared to encapsulate dehydrated ones with normal growth (Fig. 2). A similar observation was reported in Litchi (Xie et al. 2008).

Encapsulation-Dehydration

Distribution process is essentially part of succesful cryopreservation programme. Naked isolated embryogenic axes which normally used in cryopreservation studied on zygotic germplasm are unsuitable for germplasm distribution. Therefore, we reported here our initial investigations into developing suitible encapsulation-dehydration of Hevea zygotic embryos. In most cases, sucrose pretreatment alone does not dehydrate the samples to water contents sufficiently low for them to withstand freezing and rewarming, and additional physical or osmotic dehydration is required. The effect of different desiccation method was assessed by measuring survival rate and regrowth percentages of encapsulated

Table 2 The effect of two dehydration methods on the survival rate and regrowth percentages of the control (-LN) and cryopreserved $(+\mathrm{LN})$ zygotic embryos of the rubber tree treated with encapsulation dehydration protocol

\begin{tabular}{|c|c|c|c|c|c|c|}
\hline \multirow[t]{2}{*}{$\begin{array}{l}\text { Dehydration } \\
\text { method }\end{array}$} & \multirow[t]{2}{*}{$\begin{array}{l}\text { Dehydration } \\
\text { time }(\mathrm{h})\end{array}$} & \multirow[t]{2}{*}{$\begin{array}{c}\text { Moisture } \\
\text { content }(\%)\end{array}$} & \multicolumn{2}{|c|}{$+\mathrm{LN}$} & \multicolumn{2}{|c|}{$-\mathrm{LN}$} \\
\hline & & & Survival rate $(\%)$ & Regrowth (\%) & Survival rate $(\%)$ & Regrowth (\%) \\
\hline \multirow[t]{5}{*}{ silica gel } & 0 & $62.50 \pm 19.3$ & 0 & 0 & $93.73 \pm 7.6^{\mathrm{a}}$ & $90.37 \pm 9.3^{\mathrm{a}}$ \\
\hline & 3 & $33.00 \pm 17.6$ & 0 & 0 & $89.15 \pm 15.8^{\mathrm{a}}$ & $85.45 \pm 10.4^{\mathrm{a}}$ \\
\hline & 6 & $19.12 \pm 8.6$ & 0 & 0 & $85.80 \pm 12.6^{\mathrm{a}}$ & $81.00 \pm 14.3^{\mathrm{a}}$ \\
\hline & 9 & $15.74 \pm 7.5$ & 0 & 0 & $83.63 \pm 16.2^{\mathrm{a}}$ & $79.73 \pm 9.7^{\mathrm{a}}$ \\
\hline & 12 & $12.32 \pm 5.5$ & 0 & 0 & $75.87 \pm 10.8^{\mathrm{a}}$ & $71.37 \pm 12.5^{\mathrm{a}}$ \\
\hline \multirow[t]{5}{*}{ air dying } & 0 & $62.32 \pm 15.6$ & 0 & 0 & $92.00 \pm 7.9^{\mathrm{a}}$ & $89.45 \pm 9.2^{\mathrm{a}}$ \\
\hline & 2 & $35.03 \pm 12.3$ & $12.32 \pm 5.6^{\mathrm{c}}$ & $7.08 \pm 2.3^{\mathrm{c}}$ & $93.15 \pm 8.1^{\mathrm{a}}$ & $90.55 \pm 7.6^{\mathrm{a}}$ \\
\hline & 3 & $25.12 \pm 8.3$ & $27.05 \pm 7.9^{b}$ & $16.85 \pm 6.3^{\mathrm{b}}$ & $90.65 \pm 12.6^{\mathrm{a}}$ & $88.63 \pm 7.1^{\mathrm{a}}$ \\
\hline & 4 & $15.54 \pm 4.5$ & $37.50 \pm 10.6^{\mathrm{a}}$ & $27.98 \pm 7.9^{\mathrm{a}}$ & $89.75 \pm 10.9^{\mathrm{a}}$ & $83.03 \pm 8.5^{\mathrm{a}}$ \\
\hline & 5 & $12.05 \pm 7.6$ & $32.03 \pm 12.3^{\mathrm{ab}}$ & $15.05 \pm 8.6^{\mathrm{b}}$ & $87.63 \pm 7.9^{\mathrm{a}}$ & $85.72 \pm 9.1^{\mathrm{a}}$ \\
\hline
\end{tabular}

Values (mean values \pm SE) within the column of each parameter for each treatment followed by the same letter were not significantly different based on the Duncan's multiple range test $(\mathrm{P}<0.05)$ 


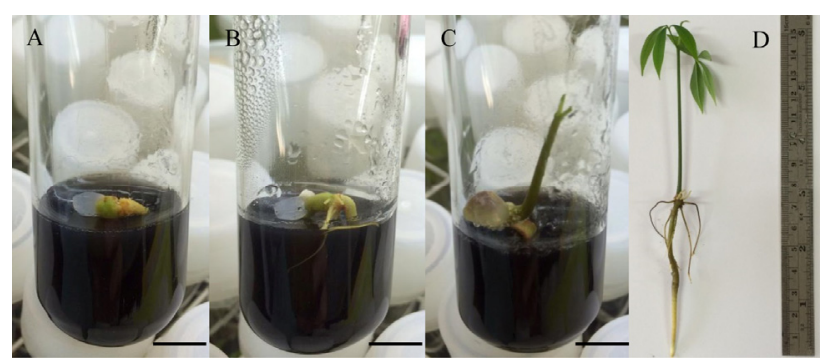

Fig. 3 A plantlet developed from an encapsulated-dehydration by air drying at 4 hours after being cryopreserved in $\mathrm{LN}\left(-196^{\circ} \mathrm{C}\right)$ at 10 days (A), 14 days (B), 21 days (C) after plating and whole plant development (D). Bar presents $1 \mathrm{~cm}$

zygotic embryos after cryopreservation.

Survival rate of cryopreserved zygotic embryos was depending on the moisture content. The maximum survival rate and regrowth percentage could be achieved only when the moisture content of embryos was reduced to $\approx 15 \%$ which dehydrated in the laminar air flow cabinet. The optimal dehydration times for encapsulated zygotic embryos were $4 \mathrm{~h}$ which gave the highest survival rate at $37.50 \%$ and regrowth percentages at $27.98 \%$ (Table 2). After cryopreserved, encapsulated zygotic embryos showed normal growth as shown in Figure 3. It was noticed that encapsulated zygotic embryos showed a relatively lower viability in comparison to naked zygotic embryos. The results obtained in this observation corroborated with the result from Normah et al. (1986). The survival rate and regrowth percentages of encapsulated zygotic embryos might be effect by the alginate bead. Since encapsulated zygotic embryos that not cryopreserved could not germinate well indicating that encapsulation matrix has inhibiting effect on the regrowth. Moreover, selecting zygotic embryos at the right developmental stage is critical importance for successful of Hevea cryopreservation experiment. Current study demonstrated that the moisture content of Hevea zygotic embryos after desiccation for $4 \mathrm{~h}$ and before immerse into $\mathrm{LN}$ was about $15 \%$. The optimum moisture content for plant germplasm before expose to $\mathrm{LN}$ is normally about $20 \%$ (Reed et al. 2006) However, once a protocol is chosen, some critical points can be adjusted to improve the plant response.

\section{Conclusion}

Zygotic embryos plays a key role in Hevea propagation and germplasm conservation programmes, as it is the only method ensuring large scale multiplication of elite clones. With this study, we clearly demonstrate that rubber tree zygotic embryo can be successfully cryopreserved using the vitrification method. Attention should be therefore given to all stages of cryopreservation procedure, since apparently minor modification can improve survival dramatically. A high tolerance to PVS2 in terms of survival and recovery was observed in Hevea zygotic embryos as incubation periods up to $120 \mathrm{~min}$ had little or no influence on these parameters. Cryopreserved storage of zygotic embryos produced from an increasing number of Hevea clones, to complement the traditional conservation of whole plants in field collection, should play a progressively more important role to ensure the safe and cost effective long term conservation of Hevea genetic resources.

\section{Acknowledgements}

This research is supported by Prince of Songkla University (Grant No. NAT560529S). The authors would like to thank the Publication Clinic, Research and Development Office, Prince of Songkla University, for technical comments and improving the manuscript.

\section{References}

Charloq, Lubis Z, Siregar TH, Damanik SB, Yazid A, Husni M (2016) Physiology changes of shelled rubber (Hevea brasilliensis Muell. Arg.) seed after 16 days storage with PEG 6000 30\% coating to induce secondary dormancy. J Agron 15:11-18

Fabre J, Dereuddre J (1990) Encapsulation-dehydration: a new approach to cryopreservation of solanum shoot tips. Cryo Letters 11:413-426

Guzmán-García E, Bradaï F, Sánchez-Romero C (2013) Cryopreservation of avocado embryogenic cultures using the dropletvitrification method. Acta Physiol Plant 35:183-193

Ighere DA, Okere A, Elizabeth J, Mary O, Olatunde F, Abiodun S (2011) In-vitro culture of Hevea brasiliensis (rubber tree) embryo. J Plant Breed Crop Sci 3:185-189

Kim HH, Cho EG, Baek HJ, Kim CY, R Joachim Keller E, Engelmann F (2004) Cryopreservation of garlic shoot tips by vitrification: effects of dehydration, rewarming, unloading and regrowth conditions. Cryo Letters 25:59-70

Kim HH, Lee YG, Shin DJ, Ko HC, Gwag JG, Cho EG, Engelmann F (2009) Development of alternative plant vitrification solutions in droplet-vitrification procedures. Cryo Letters 30:320-334

Martinez MT, Ballester A, Vieitez AM (2003) Cryopreservation of embryogenic cultures of Quercus robur using desiccation and vitrification procedures. Cryobiology 46:182-189

Nishizawa S, Sakai A, Amano Y, Matsuzawa T (1993) Cryopreservation of asparagus (Asparagus officinalis L.) embryogenic suspension cells and subsequent plant regeneration by vitrification. Plant Sci 91:67-73 
Normah MN, Chin HF, Hor YL (1986) Desiccation and cryopreservation of embryonic axes of Hevea brasiliensis Muell. Arg. Pertanika 9:299-303

Panis B, Piette B, Swennen R (2005) Droplet vitrification of apical meristems: a cryopreservation protocol applicable to all Musaceae. Plant Sci 168:45-55

Peran R, Berjak P, Pammenter NW, Kioko JI (2006) Cryopreservation, encapsulation and promotion of shoot production of embryonic axes of a recalcitrant species Ekebergia capensis, Sparrm. Cryo Letters 27:5-16

Reed BM, Schumachera L, Wanga N, Achinoa J, Barker RE (2006) Cryopreservation of bermudagrass Germplasm by encapsulation dehydration. Crop Sci 46:4-11

Sajini KK, Karun A, Amarnath CH, Engelmann F (2011) Cryopreservation of coconut (Cocos nucifera L.) zygotic embryos by vitrification. Cryo Letters 32:317-328

Sakai A, Engelmann F (2007) Vitrification, encapsulation-vitrification and droplet-vitrification: a review. Cryo Letters 28:151-172

Sakai A, Kobayashi S, Oiyama I (1990) Cryopreservation of nucellar cells of navel orange (Citrus sinensis Osb. var. brasiliensis Tanaka) by vitrification. Plant Cell Rep 9:30-33

Sakai A, Kobayashi S, Oiyama I (1991) Cryopreservation of nucellar cells of navel orange (Citrus sinensis Osb.) by a simple freezing method. Plant Sci 74:243-248

Sam YY (1999) Cryopreservation of rubber (Hevea brasiliensis) zygotic embryos using vitrification technique. Master of Agriculture Science, Faculty of Agriculture, Putra Malaysia University
Sant R, Panis B, Taylor M, Tyagi A (2008) Cryopreservation of shoot-tips by droplet vitrification applicable to all taro (Colocasia esculenta var. esculenta) accessions. Plant Cell Tissue Organ Cult 92:107-111

Uemura M, Steponkus PL (2003) Modification of the intracellular sugar content alters the incidence of freeze-induced membrane lesions of protoplasts isolated from Arabidopsis thaliana leaves. Plant Cell Environ 26:1083-1096

Wang YL, Fan MJ, Liaw SI (2005) Cryopreservation of in vitro-grown shoot tips of papaya (Carica papaya L.) by vitrification. Bot Bull Acad Sin 46:29-34

Xie YM, Zeng JW, Zhang QM, Yi GJ (2008) Cryopreservation of litchi embryogenic suspension cells by vitrification technique. (In Chinese with English abstract). Chin J Trop Crops 29: 622-625

Yap LV, Hor YL, Normah MN (1998) Effect of sucrose preculture and subsequent desiccation on cryopreservation of alginateencapsulated Hevea brasiliensis embryo. Proc. of the IUFRO Seed Symposium 1998, Kuala Lumpur, Malaysia. pp 140-145

Yap LV, Noor NM, Clyde MM, Chin HF (2011) Cryopreservation of Garcinia cowa shoot tips by vitrification: the effects of sucrose preculture and loading treatment on ultrastructural changes in meristematic cells. Cryo Letters 32:188-196

Zhou QN, Sun AH, Li Z, Hua YW, Jiang ZH, Huang TD, Dai XM, Huang HS (2012) Cryopreservation and plant regeneration of anther callus in Hevea by vitrification. Afr $\mathrm{J}$ Biotechnol 11:7212-7217 